\title{
Measurement of time-integrated $D^{0} \rightarrow h h$ asymmetries at $\mathrm{LHCb}$
}

\author{
Pietro Marino* \\ Scuola Normale Superiore and INFN-Pisa \\ E-mail: pietro.marino@sns.it
}

LHCb collected the world's largest sample of charm decays during LHC Run I, corresponding to an integrated luminosity of $3 \mathrm{fb}^{-1}$. This has permitted many precision measurements of charm mixing and $\mathrm{CP}$ violation parameters. One of the most precise and important observables is the so-called $\triangle A_{C P}$ parameter, corresponding to the difference between the time-integrated $C P$ asymmetry in singly Cabibbo-suppressed $D^{0} \rightarrow K^{+} K^{-}$and $D^{0} \rightarrow \pi^{+} \pi^{-}$decay modes. The flavour of the $D^{0}$ meson is inferred from the charge of the pion in $D^{*+} \rightarrow D^{0} \pi^{+}$and $D^{*-} \rightarrow \bar{D}^{0} \pi^{-}$decays. $\Delta A_{C P} \equiv A_{\text {raw }}\left(K^{+} K^{-}\right)-A_{\text {raw }}\left(\pi^{+} \pi^{-}\right)$is measured to be $\Delta A_{C P}=(-0.10 \pm 0.08 \pm 0.03) \%$, where the first uncertainty is statistical and the second systematic. The measurement is consistent with the no- $C P$-violation hypothesis and represents the most precise measurement of time-integrated $C P$ asymmetry in the charm sector.

16th International Conference on B-Physics at Frontier Machines 2-6 May 2016

Marseille, France

* Speaker.

${ }^{\dagger}$ On behalf of the LHCb Collaboration 


\section{Introduction}

All direct measurements of elementary particle phenomena to date support the CKM phase, within the current theoretical and experimental uncertainties, being the only source of $C P$ violation. Charm is a particularly good sector to probe deviations from the Standard Model (SM), since the amount of $C P$ violation is expected to be below the percent level $[1,2,3]$, but large theoretical uncertainties due to long distance interactions prevent precise SM calculations. On the other hand, charm hadrons provide a unique opportunity to search for $C P$ violation with particles containing only up-type quarks.

The LHCb detector [4] is a single-arm forward spectrometer covering the pseudorapidity range $2<\eta<5$, designed for the study of particles containing $b$ or $c$ quarks. The detector is composed of a silicon-strip vertex detector surrounding the $p p$ interaction region that allows $c$-and $b$-hadrons to be identified from their typically long flight distance, and a tracking system that provides a measurement of momentum of charged particles. In addition, two ring-imaging Cherenkov detectors are present to discriminate between different species of charged hadrons. An electromagnetic and hadron calorimeter complete the detector, located upstream of muon stations.

\section{2. $C P$ violation in two-body $D^{0}$ decays}

Time-integrated $C P$ violation in the decay $D^{0} \rightarrow f$, where $f=\left\{K^{+} K^{-}, \pi^{+} \pi^{-}\right\}$, can be parametrised as the difference in the decay rates, $\Gamma$ :

$$
A_{C P}(f)=\frac{\Gamma\left(D^{0} \rightarrow f\right)-\Gamma\left(\bar{D}^{0} \rightarrow f\right)}{\Gamma\left(D^{0} \rightarrow f\right)+\Gamma\left(\bar{D}^{0} \rightarrow f\right)} .
$$

$D^{* \pm}$, decaying strongly to $D^{0} / \bar{D}^{0}$, are used to "tag" the flavour of $D^{0}$ mesons. Experimentally, the raw asymmetry is measured by counting the number of reconstructed $D^{*+}$ and $D^{*-}$ decays:

$$
\begin{aligned}
A_{\text {raw }}(f) & =\frac{N\left(D^{*+} \rightarrow D^{0}(\rightarrow f) \pi^{+}\right)-N\left(D^{*-} \rightarrow \bar{D}^{0}(\rightarrow f) \pi^{-}\right)}{N\left(D^{*+} \rightarrow D^{0}(\rightarrow f) \pi^{+}\right)+N\left(D^{*-} \rightarrow \bar{D}^{0}(\rightarrow f) \pi^{-}\right)} \\
& \approx A_{C P}(f)+A_{\operatorname{det}}(\pi)+A_{\text {prod }}\left(D^{*+}\right),
\end{aligned}
$$

where $A_{\text {det }}(\pi)$ is the detection asymmetry for the tagging pion and $A_{\text {prod }}\left(D^{*+}\right)$ is the production asymmetry for the $D^{*+}$. Measuring the absolute $C P$ asymmetries $A_{C P}(f)$ with a sensitivity of $\mathscr{O}\left(10^{-3}\right)$ is experimentally challenging, since $A_{\operatorname{det}}(\pi)$ and $A_{\text {prod }}\left(D^{*+}\right)$ (both about $1 \%$ ) have to be known with the same level of precision. However, such a precision can be reached by the $\triangle A_{C P}$ observable, which is defined as the difference between the $C P$ asymmetry of the $D^{0} \rightarrow K^{+} K^{-}$ decays and the $D^{0} \rightarrow \pi^{+} \pi^{-}$decays:

$$
\Delta A_{C P} \equiv A_{C P}\left(K^{+} K^{-}\right)-A_{C P}\left(\pi^{+} \pi^{-}\right) \approx A_{\text {raw }}\left(K^{+} K^{-}\right)-A_{\text {raw }}\left(\pi^{+} \pi^{-}\right) .
$$

In the $\Delta A_{C P}$ observable, the $D^{*+}$ production asymmetry and the detection asymmetry of tagging pions cancel out in the difference.

In the full Run I data sample of $\mathrm{LHCb}$, corresponding to an integrated luminosity of about $3 \mathrm{fb}^{-1}, 7.7 \times 10^{6} D^{0} \rightarrow K^{+} K^{-}$decays and $2.5 \times 10^{6} D^{0} \rightarrow \pi^{+} \pi^{-}$are reconstructed; the $D^{*+}-D^{0}$ mass difference distributions are shown in Fig. 1. The measured value of $\Delta A_{C P}$ is found to be [5]: 

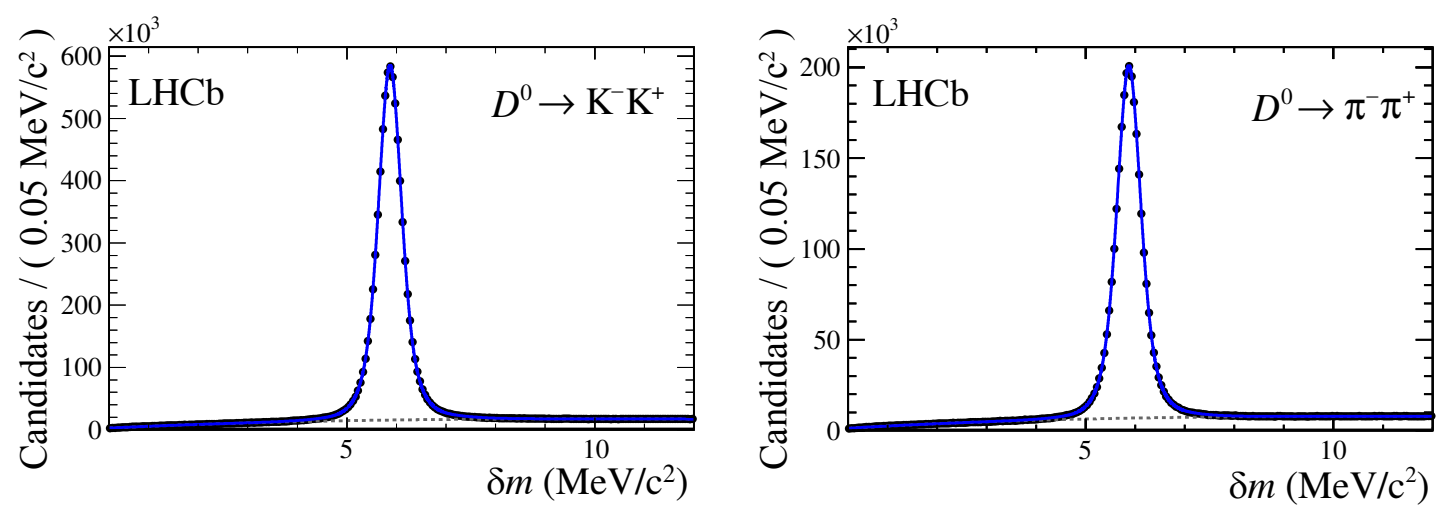

Figure 1: $\Delta m \equiv m\left(D^{*+}\right)-m\left(D^{0}\right)$ distribution for $D^{0} \rightarrow K^{+} K^{-}$decays (left) and for $D^{0} \rightarrow \pi^{+} \pi^{-}$decays (right).

$$
\Delta A_{C P}=(-0.10 \pm 0.08(\text { stat }) \pm 0.03(\text { syst })) \%
$$

The $0.03 \%$ systematic error comes from sources such as uncertainties in secondary decays, misreconstructed decays and fiducial cuts. The result is compatible with the no- $C P$-violation hypothesis and represents the most precise determination to date of this observable. To check for possible reconstruction biases, the stability of $\Delta A_{C P}$ is investigated as a function of many reconstructed quantities, such as the number of reconstructed primary vertices, the $D^{0}$ and tagging pion transverse momenta, the $D^{0}$ flight distance, the quality of the $D^{0}$ vertex and particle identification variables. No significant dependence is found on any quantity.

\section{Conclusion}

The large sample of $D$-meson decays collected by the LHCb experiment in Run I allows us to reach sensitivities close to the SM expectation of $C P$ violation in the charm sector. In the case of the $\Delta A_{C P}$ measurement the sensitivity is already below $\mathscr{O}\left(10^{-3}\right)$ but no-hint of $C P$ violation has been found so far. In the current Run II and in the future LHCb-Upgrade programme, the search for $C P$ violation will continue with an increasing precision. The dynamics of charm decays will be studied with unprecedented statistics, increasing our knowledge of the SM and our sensitivity to probe virtual contributions from new particles beyond it.

\section{References}

[1] H. Y. Cheng and C. W. Chiang, "SU(3) symmetry breaking and CP violation in $D \rightarrow P P$ decays," Phys. Rev. D 86, 014014 (2012) [arXiv:1205.0580 [hep-ph]].

[2] H. n. Li, C. D. Lu and F. S. Yu, "Branching ratios and direct CP asymmetries in $D \rightarrow P P$ decays," Phys. Rev. D 86, 036012 (2012) [arXiv:1203.3120 [hep-ph]].

[3] J. Brod, Y. Grossman, A. L. Kagan and J. Zupan, "A Consistent Picture for Large Penguins in $D \rightarrow \pi^{+} \pi^{-}, K^{+} K^{-}$, JHEP 1210, 161 (2012) [arXiv:1203.6659 [hep-ph]].

[4] A. A. Alves, Jr. et al. [LHCb Collaboration] The LHCb Detector at the LHC, JINST3, S08005 (2008). 
[5] R. Aaij et al. [LHCb Collaboration], "Measurement of the difference of time-integrated CP asymmetries in $D^{0} \rightarrow K^{-} K^{+}$and $D^{0} \rightarrow \pi^{-} \pi^{+}$decays," Phys. Rev. Lett. 116, no. 19, 191601 (2016) [arXiv:1602.03160 [hep-ex]]. 